\title{
Body Mass Index, Physical Activity, and Fracture Among Young Adults: Longitudinal Results From the Thai Cohort Study
}

\author{
Susan Jordan ${ }^{1,2}$, Lynette Lim $^{3}$, Janneke Berecki-Gisolf ${ }^{4}$, Chris Bain ${ }^{2,3}$, Sam-ang Seubsman ${ }^{3,5}$ \\ Adrian Sleigh ${ }^{3}$, Emily Banks ${ }^{3}$, and the Thai Cohort Team* \\ ${ }^{1}$ School of Population Health, University of Queensland, Brisbane, Australia \\ ${ }^{2}$ Population Health Department, The Queensland Institute of Medical Research, Brisbane, Australia \\ ${ }^{3}$ National Centre for Epidemiology and Population Health, ANU College of Medicine, Biology and the Environment, \\ The Australian National University, Canberra, Australia \\ ${ }^{4}$ Monash Injury Research Institute, Monash University, Melbourne, Australia \\ ${ }^{5}$ Thai Health-Risk Transition Study, School of Human Ecology, Sukhothai Thammathirat Open University, Nonthaburi, Thailand
}

Received December 10, 2012; accepted July 9, 2013; released online September 28, 2013

Copyright $(\subset) 2013$ Susan Jordan et al. This is an open access article distributed under the terms of Creative Commons Attribution License, which permits unrestricted use, distribution, and reproduction in any medium, provided the original author and source are credited.

\begin{abstract}
Background: We investigated risk factors for fracture among young adults, particularly body mass index (BMI) and physical activity, which although associated with fracture in older populations have rarely been investigated in younger people.

Methods: In 2009, 4 years after initial recruitment, 58204 Thais aged 19 to 49 years were asked to self-report fractures incident in the preceding 4 years. Conditional logistic regression was used to calculate odds ratios (ORs) and $95 \%$ CIs for associations of fracture incidence with baseline BMI and physical activity.

Results: Very obese women had a $70 \%$ increase in fracture risk (OR $=1.73,95 \%$ CI $1.21-2.46)$ as compared with women with a normal BMI. Fracture risk increased by $15 \%$ with every $5-\mathrm{kg} / \mathrm{m}^{2}$ increase in BMI. The effects were strongest for fractures of the lower limbs. Frequent purposeful physical activity was also associated with increased fracture risk among women ( $\mathrm{OR}=1.52,95 \%$ CI 1.12-2.06 for 15 episodes/week vs none). Neither BMI nor physical activity was associated with fracture among men, although fracture risk decreased by $4 \%$ with every additional 2 hours of average sitting time per day $(\mathrm{OR}=0.96,95 \%$ CI $0.93-0.99)$.

Conclusions: The increase in obesity prevalence will likely increase fracture burden among young women but not young men. While active lifestyles have health benefits, our results highlight the importance of promoting injury prevention practices in conjunction with physical activity recommendations, particularly among women.
\end{abstract}

Key words: fracture; obesity; body size; physical activity; prevention; epidemiology; young adults

\section{INTRODUCTION}

Fractures are an important cause of morbidity among young adults. They can result in prolonged absence from work, considerable health-resource use, and frequent long-term disability. ${ }^{1}$ They are particularly important in developing countries with young populations where injury accounts for a large proportion of the disease burden. ${ }^{2}$ Despite this, few studies have investigated risk factors for fracture among adults younger than 50 years. One research group has investigated the effects of dietary calcium, ${ }^{3}$ serum vitamin $\mathrm{D},{ }^{4}$ and levels of sex steroid hormones, ${ }^{5}$ but other exposures were not examined. Thus an evidence base for targeted prevention is lacking.

Low body mass index (BMI) and low physical activity level are 2 potentially modifiable factors that have been consistently associated with fracture risk among postmenopausal women and older men. ${ }^{6,7}$ However, it is unclear whether these factors

Address for correspondence. Dr Susan Jordan, The Queensland Institute of Medical Research, Locked Bag 2000, PO Royal Brisbane Hospital, Brisbane 4029, Australia (e-mail: susan.jordan@qimr.edu.au).

*The Thai Cohort Study Team consists of Jaruwan Chokhanapitak, Chaiyun Churewong, Suttanit Hounthasarn, Suwanee Khamman, Daoruang Pandee, Suttinan Pangsap, Tippawan Prapamontol, Janya Puengson, Yodyiam Sangrattanakul, Sam-ang Seubsman, Boonchai Somboonsook, Nintita Sripaiboonkij, Pathumvadee Somsamai, Duangkae Vilainerun, Wanee Wimonwattanaphan (Thailand); and Chris Bain, Emily Banks, Cathy Banwell, Bruce Caldwell, Gordon Carmichael, Tarie Dellora, Jane Dixon, Sharon Friel, David Harley, Matthew Kelly, Tord Kjellstrom, Lynette Lim, Anthony McMichael, Tanya Mark, Adrian Sleigh, Lyndall Strazdins, Vasoontara Yiengprugsawan (Australia). 
have an effect on fracture risk among men and women during early and mid-adulthood.

Some studies of younger adults investigated the effects of BMI and physical activity on bone mineral density (BMD), a proxy for future fracture risk. Most found that BMD increases with weight ${ }^{8-13}$ and physical activity. ${ }^{9,14,15}$ However, this may not equate directly to fracture risk, as higher fat mass relative to lean body mass can adversely affect bone strength, ${ }^{16-20}$ fat mass may increase instability and propensity to fall, ${ }^{21}$ and the increased bone density associated with greater weight may not fully compensate for the extra load on bones. ${ }^{22}$ Furthermore, while physical activity may increase bone strength, ${ }^{7}$ it may also increase exposure to situations that increase fracture risk. Therefore it is important to examine fracture outcomes rather than proxies of such outcomes.

Obesity and physical inactivity are major health concerns in Western countries and emerging concerns in newly industrialized countries. ${ }^{23}$ We are therefore interested to see how BMI (as a measure of obesity) and physical activity influence actual fracture incidence among younger people. This will help us understand how increased BMI among younger people will affect the population burden of fracture and how uptake of physical activity recommendations could modify this relationship. We have used longitudinal data from a large study of young Thai adults to investigate these relationships in detail.

\section{METHODS}

The Thai Cohort Study was established to examine the health consequences of Thailand's rapid socioeconomic development. The methods have been reported previously. ${ }^{24}$ In brief, participants were recruited from the student body of Sukhothai Thammathirat Open University (STOU). STOU students reside all over Thailand and have a modest socioeconomic status. Many are rural dwellers and most work full-time. In 2005 all STOU students who had completed at least 1 semester of study (approximately 200000) were invited to participate. Overall, 87134 students (47314 women and 39820 men) completed questionnaires (44\%).

Baseline measures included demographic variables (age, sex, area of residence, income, and prior education), lifestyle variables (smoking, alcohol, and physical activity), medical diagnoses (diabetes, ischemic heart disease, kidney disease, thyroid disease, and cancer) and self-reported height and weight. We calculated BMI as weight $(\mathrm{kg}) /$ height $(\mathrm{m})^{2}$ and categorized this using suggested cut-points for overweight and obesity among Asian populations. ${ }^{25}$ We also assessed the effect of height on fracture risk because some studies have suggested that height could be associated with some fracture types. ${ }^{26-28}$ The number of episodes of physical activity per week was calculated by summing responses to questions on number of episodes of walking (at work, home, or for exercise), plus episodes of mild (eg, Tai Chi), moderate (eg, cycling at a regular pace, carrying light loads), and strenuous (eg, heavy lifting, digging, running) exercise. Participants were not asked to exclude exercise that was done during their work. We also considered separately the effect of frequency of housework/gardening (seldom/never; 1-3 times/month; 1-2 times/week; 3-4 times/week; and most days), as this provided discriminating estimates of physical activity in previous analyses. ${ }^{29}$ Sitting time was ascertained by the question, "How many hours per day do you usually spend sitting for any purpose (eg, reading, resting, working, thinking)?”.

In 2009, a 4-year follow-up mail survey was conducted. Of the initial 87134 participants, 289 died before the follow-up survey, 20 withdrew from the study, and an additional 1608 had no contact details or insufficient information on their identity. A follow-up survey was mailed to 85217 . After 4 rounds of mail-outs and more than 80000 telephone calls, there were 10207 for whom we still could not confirm an address. A further 14441 did not return their survey, and 60569 participants replied (71.1\% of the 85217 approached). Of these, $55 \%$ were women. Median age was 34 years (range 19-92).

Using the question, "In your life have you ever experienced a fracture to the areas of your body mentioned below?", participants were asked to indicate whether they had experienced a fracture at any of 13 specified sites (finger/ toe; wrist; arm; collarbone; rib; skull; face/jaw/nose; neck; back; pelvis; leg; ankle; other) during their lifetime and the age at which they sustained the fracture. Fractures reported to have occurred at an age greater than the participant's age at baseline were considered to be incident fractures. Variables related to women's contraceptive use were also taken from the 2009 survey.

\section{Statistical analyses}

Men and women were considered separately in the analyses, due to the possibility that fracture etiology might vary by sex. We decided a priori to exclude adults older than 50 years because our interest was the effect of BMI and physical activity on younger adults. Furthermore, very few of our participants (approximately 3\%) were in that older age group and, while we considered the possibility that associations might vary by menopausal status, we lacked the statistical power to test for such an interaction. In addition we excluded those with a cancer diagnosis $(n=371)$ because we felt that adjusting for this might not adequately account for the broad spectrum of effects of different cancers on BMI and fracture occurrence. Skull fractures may have been over-reported (due to confusion with head trauma more generally), so these were also excluded, as were events for which fracture information was missing $(n=21)$. In site-specific analyses we grouped wrist and arm fractures (upper limb) and leg and ankle fractures (lower limb).

Participants were asked to record the age at which their fracture(s) was sustained rather than the date, because age at 
fracture is far more likely to be recalled than the date of a fracture. Thus our information was not precise enough to undertake a survival-type analysis. Univariate analyses stratified by age group (10-year bands) suggested that associations between fracture and $\mathrm{BMI} /$ physical activity persisted across all ages, but we were concerned that adjusting for age in our analyses might not be sufficient given the strong relationships of increasing age with both BMI and fracture. As a result we have undertaken all multivariable analyses using conditional logistic regression, stratified by age in years, to calculate odds ratios (ORs) and 95\% CIs as estimates of relative risk. All analyses were adjusted for history of fracture before 2005 (yes/no), income ( $\leq 7000$, $>7000-10000,>10000-20000,>20000$ Thai Baht), smoking status (ever/never), ever-use of alcohol (yes/no), vascular disease (ischemic heart disease or stroke; yes/no). Analyses considering BMI were adjusted for episodes of purposeful physical activity per week $(0,1-5,6-10,11-15,>15)$, and analyses considering physical activity were adjusted for BMI $\left(<18.5,18.5\right.$ to $<23,23$ to $<24.5,25$ to $\left.<30, \geq 30.0 \mathrm{~kg} / \mathrm{m}^{2}\right)$. For women, analyses were additionally adjusted for ever-use of depot medroxyprogesterone acetate (DMPA). Factors such as parity, prior education, and place of residence, which did not materially change the estimates, were excluded from the final model. To assess trends of risk with increasing exposure, the continuous forms of the variables divided by the interval of interest (ie, $10 \mathrm{~cm}$ for height; $5 \mathrm{~kg} / \mathrm{m}^{2}$ for BMI; per 5 episodes for physical activity; per 2 hours of sitting time) were used in the models. For amount of housework/gardening the categorical variable was entered into the model as a linear term and its significance assessed. All analyses were done using SAS statistical software (version 9.2, SAS Institute Inc., Cary, NC, USA).

Ethics approval was obtained from the STOU Research and Development Institute (protocol 0522/10) and the Australian National University Human Research Ethics Committee (protocol 2004344). Informed written consent was obtained from all participants.

\section{RESULTS}

Our analyses included 32339 women and 25865 men younger than 50 who completed the survey in 2009. Of these, $902(2.8 \%)$ women and 1168 (4.5\%) men reported having had a fracture since 2005. Participant characteristics are summarized in Table 1. Fracture was more common among older women than among younger women, whereas the opposite trend was seen among men $(P<0.0001$ respectively). For both sexes those who smoked or had vascular disease or a fracture before 2005 were significantly more likely to have had a fracture since 2005. Significant associations were also seen for BMI and frequency of physical activity. For women an association was also seen for ever use of DMPA contraceptives; while for men, income, prior education, and ever-use of alcohol were also significantly associated with fracture incidence.

Table 2 shows the results of multivariable analyses of the associations of fracture incidence with BMI and height. Women in the highest category of obesity had a $70 \%$ higher risk of fracture as compared with those with normal BMI values (OR 1.73, 95\% CI 1.21-2.46), and risk increased by $15 \%$ for every additional $5-\mathrm{kg} / \mathrm{m}^{2}$ increase in BMI. Being underweight did not increase fracture risk. For men there was no linear association between BMI and fracture after adjustment for age, although underweight men had a significant $26 \%$ decrease in risk as compared with men in the normal BMI range. The interaction term for sex and BMI was significant $(P=0.002)$. There was a suggestion that taller men and taller women had an increased fracture risk as compared with shorter participants, but the effect was not statistically significant.

Associations between physical activity measures and fracture risk are shown in Table 3. Among women fracture risk increased with increasing episodes of purposeful physical activity (excluding gardening and housework) $(\mathrm{OR}=1.52$, 95\% CI 1.12-2.06 for $>15$ episodes per week vs none), but no significant relationship was observed among men $(P($ interaction $)=0.06)$. Conversely, more sitting time (adjusted for episodes of physical activity) modestly decreased fracture risk among men (OR 0.96, 95\% CI 0.93-0.99 per additional 2 hours of sitting time per day) but not women, although the interaction term was not significant $(P=0.4)$. There was no significant association of frequency of household chores or manual worker status (versus those who reported being professionals, office workers, or managers) with fracture.

We stratified our analyses to test if the association between BMI and fracture among women was modified by the frequency of physical activity (in categories of frequency) but found no evidence of such an effect $(P($ interaction $)=0.7)$.

Table 4 shows the effects of BMI, number of episodes of purposeful physical activity, and hours of sitting time on site-specific fracture risk among women and men. Among women, obesity was most strongly associated with lower limb fracture (ankle and leg), with a significant 32\% increase in fracture risk per 5-unit increase in BMI. The risk of upper limb fracture was nonsignificantly elevated among very obese women. There were no clear patterns of association between BMI and site-specific fracture risk among men. There was also a suggestion that more-frequent purposeful physical activity modestly increased fracture risk at all sites among women, although the estimates were not statistically significant for upper or lower limb fractures. Purposeful physical activity was not clearly associated with fracture at any site among men. As for the main results, each additional 2 hours of sitting time per day reduced the risk of lower limb fracture by $6 \%$ (OR $0.94,95 \%$ CI $0.88-0.99$ ) among men. 
Table 1. Baseline characteristics of women and men in the Thai Cohort Study (2005-2009), according to self-report of fracture since 2005

\begin{tabular}{|c|c|c|c|c|c|c|}
\hline & \multicolumn{2}{|c|}{ Women - Fracture } & \multirow[b]{2}{*}{$P$-value ${ }^{a}$} & \multicolumn{2}{|c|}{ Men - Fracture } & \multirow[b]{2}{*}{$P$-value ${ }^{\mathrm{a}}$} \\
\hline & $\begin{array}{c}\text { Yes } \\
(n=902)\end{array}$ & $\begin{array}{c}\text { No } \\
(n=31437)\end{array}$ & & $\begin{array}{c}\text { Yes } \\
(n=1168)\end{array}$ & $\begin{aligned} & \text { No } \\
(n= & 24697)\end{aligned}$ & \\
\hline & \multicolumn{2}{|c|}{ Mean (SD) } & & \multicolumn{2}{|c|}{ Mean (SD) } & \\
\hline Age (yrs) & $30.9(7.5)$ & $29.7(7.1)$ & $<0.0001$ & $31.5(7.3)$ & $32.5(7.6)$ & $<0.0001$ \\
\hline \multirow[t]{2}{*}{ Height $(\mathrm{cm})$} & $158.0(5.8)$ & $157.5(5.5)$ & 0.01 & $168.7(5.9)$ & $168.3(5.9)$ & 0.02 \\
\hline & \multicolumn{2}{|c|}{$n^{\mathrm{b}}(\%)$} & & \multicolumn{2}{|c|}{$n^{\mathrm{b}}(\%)$} & \\
\hline \multicolumn{7}{|l|}{ Income (Thai baht) } \\
\hline$\leq 7000$ & $366(41.5)$ & $13664(44.5)$ & 0.3 & 389 (33.7) & 7759 (32.1) & $<0.0001$ \\
\hline$>7000-10000$ & $218(24.7)$ & 7419 (24.2) & & $323(28.0)$ & $5660(23.4)$ & \\
\hline$>10000-20000$ & $222(25.2)$ & 7020 (22.9) & & $319(27.6)$ & 7560 (31.2) & \\
\hline$>20000$ & $76(8.6)$ & $2623(8.5)$ & & $123(10.7)$ & 3229 (13.3) & \\
\hline \multicolumn{7}{|l|}{ Prior education } \\
\hline Junior high & $22(2.5)$ & $603(2.1)$ & 0.1 & $46(4.0)$ & $1110(4.5)$ & 0.03 \\
\hline High school & $378(42.1)$ & $12173(37.7)$ & & $588(50.4)$ & $11885(47.8)$ & \\
\hline Diploma/certificate & $253(28.1)$ & 9632 (30.7) & & $288(24.7)$ & $5658(23.0)$ & \\
\hline University & $246(27.4)$ & $8953(28.6)$ & & $244(20.9)$ & $5993(24.3)$ & \\
\hline \multicolumn{7}{|l|}{ Place of residence } \\
\hline City & $487(54.4)$ & $16221(51.9)$ & 0.2 & $596(51.2)$ & $12121(49.4)$ & 0.7 \\
\hline Countryside & 409 (45.7) & $15011(48.1)$ & & $568(48.8)$ & $12413(50.6)$ & \\
\hline \multicolumn{7}{|l|}{ Parity } \\
\hline 0 & $543(61.8)$ & 20164 (65.6) & 0.07 & & & \\
\hline 1 & 166 (18.9) & $5216(17.0)$ & & & & \\
\hline 2 & $135(15.4)$ & $4449(14.1)$ & & & & \\
\hline$\geq 3$ & $35(4.0)$ & $923(3.0)$ & & & & \\
\hline \multicolumn{7}{|l|}{ Body mass index } \\
\hline$<18.5$ & $156(17.3)$ & $6434(20.5)$ & $<0.0001$ & $57(4.9)$ & $1407(5.7)$ & 0.04 \\
\hline $18.5-<23.0$ & $528(58.4)$ & $18423(58.6)$ & & $610(52.2)$ & $11758(47.6)$ & \\
\hline $23.0-<25.0$ & $85(9.4)$ & 3127 (10.0) & & $239(20.5)$ & $5443(22.0)$ & \\
\hline $25.0-<30.0$ & $88(9.8)$ & $2473(7.9)$ & & $220(18.8)$ & $4972(20.1)$ & \\
\hline$\geq 30.00$ & $36(4.0)$ & $641(2.0)$ & & $28(2.4)$ & $754(3.1)$ & \\
\hline \multicolumn{7}{|l|}{$\begin{array}{l}\text { Physical activity } \\
\text { (episodes/week) }\end{array}$} \\
\hline 0 & $72(8.0)$ & $3028(9.6)$ & 0.002 & $58(5.0)$ & $1429(5.8)$ & 0.002 \\
\hline $1-5$ & $228(25.3)$ & $9329(29.7)$ & & $248(21.2)$ & $5473(22.2)$ & \\
\hline $6-10$ & $323(35.8)$ & $10743(34.2)$ & & $327(28.0)$ & 7567 (30.7) & \\
\hline $11-15$ & $144(16.0)$ & 4416 (14.1) & & $285(24.4)$ & $4786(19.4)$ & \\
\hline$>15$ & $117(13.0)$ & $3250(10.3)$ & & 232 (19.9) & $4968(20.1)$ & \\
\hline Missing & $18(2.0)$ & $669(2.1)$ & & $18(1.5)$ & $469(1.9)$ & \\
\hline \multicolumn{7}{|l|}{ Smoking } \\
\hline Ever & $67(7.7)$ & $1587(5.2)$ & 0.001 & $642(56.3)$ & $12669(52.4)$ & 0.01 \\
\hline Never & $805(92.3)$ & 28947 (94.8) & & 499 (43.7) & $11525(47.6)$ & \\
\hline \multicolumn{7}{|l|}{ Alcohol consumption } \\
\hline Current & $468(52.5)$ & $16283(52.5)$ & 0.9 & $945(81.6)$ & $19280(78.8)$ & 0.02 \\
\hline None & $423(47.5)$ & $14727(47.5)$ & & $213(18.4)$ & $5182(21.2)$ & \\
\hline Ever used DMPA ${ }^{c}$ & & & & & & \\
\hline Yes & $169(18.7)$ & 4733 (15.1) & 0.002 & & & \\
\hline No & $733(81.3)$ & $26690(84.9)$ & & & & \\
\hline Thyroid disease & & & & & & \\
\hline Yes & $45(5.0)$ & 1666 (5.3) & 0.7 & $20(1.7)$ & $336(1.4)$ & 0.3 \\
\hline No & $857(95.0)$ & $29771(94.7)$ & & $1148(98.3)$ & $24360(98.6)$ & \\
\hline Kidney disease & & & & & & \\
\hline Yes & $32(3.6)$ & $796(2.5)$ & 0.06 & $38(3.3)$ & $605(2.5)$ & 0.08 \\
\hline No & $870(96.5)$ & 30641 (97.5) & & $1130(96.8)$ & 24091 (97.6) & \\
\hline Vascular disease & & & & & & \\
\hline Yes & $10(1.1)$ & $134(0.4)$ & 0.002 & $13(1.1)$ & $139(0.6)$ & 0.02 \\
\hline No & 892 (98.9) & 31303 (99.6) & & 1155 (98.9) & 24557 (99.4) & \\
\hline Fracture before 2005 & & & & & & \\
\hline Yes & $201(22.3)$ & $4282(13.6)$ & $<0.0001$ & $427(36.6)$ & 7088 (28.7) & $<0.0001$ \\
\hline No & 701 (77.7) & 27155 (86.4) & & 741 (63.4) & 17609 (71.3) & \\
\hline
\end{tabular}

${ }^{a} P$-values are from $t$-test for continuous variables and $\chi^{2}$ tests for categorical variables. $P<0.05$ is considered significant.

${ }^{b}$ Numbers may not sum to total because of missing data.

'DMPA = depot medroxyprogesterone acetate. 
Table 2. Associations of body mass index (BMI) and height with fracture incidence among 32339 women and 25865 men in the Thai Cohort Study (2005-2009)

\begin{tabular}{|c|c|c|c|c|c|c|c|c|}
\hline & \multicolumn{4}{|c|}{ Women } & \multicolumn{4}{|c|}{ Men } \\
\hline & $\begin{array}{c}\text { Total } \\
n^{\text {a }}\end{array}$ & $\begin{array}{c}\text { Fracture } \\
n(\%)\end{array}$ & OR $95 \% \mathrm{Cl}^{\mathrm{b}}$ & OR $95 \% \mathrm{Cl}^{\mathrm{c}, \mathrm{d}}$ & $\begin{array}{c}\text { Total } \\
n^{\mathrm{a}}\end{array}$ & $\begin{array}{c}\text { Fracture } \\
n(\%)\end{array}$ & OR $95 \% \mathrm{Cl}^{\mathrm{b}}$ & OR $95 \% \mathrm{Cl}^{\mathrm{C}}$ \\
\hline \multicolumn{9}{|l|}{ BMI } \\
\hline$<18.5$ & 6590 & $156(2.4)$ & $0.93(0.77-1.12)$ & $0.94(0.78-1.14)$ & 1464 & 57 (3.9) & $0.72(0.54-0.96)$ & $0.74(0.56-0.99)$ \\
\hline $18.5-<23.0$ & 18951 & $528(2.8)$ & 1.00 & 1.00 & 12368 & $610(4.9)$ & 1.00 & 1.00 \\
\hline $23.0-<25.0$ & 3212 & $85(2.7)$ & $0.91(0.71-1.15)$ & $0.90(0.71-1.14)$ & 5682 & $239(4.2)$ & $0.92(0.79-1.07)$ & $0.92(0.79-1.07)$ \\
\hline $25.0-<30.0$ & 2561 & $88(3.4)$ & $1.16(0.91-1.48)$ & $1.11(0.87-1.41)$ & 5192 & $220(4.2)$ & $0.94(0.80-1.10)$ & $0.93(0.79-1.09)$ \\
\hline$\geq 30.00$ & 677 & $36(5.3)$ & $1.82(1.27-2.62)$ & $1.73(1.21-2.46)$ & 782 & $28(3.6)$ & $0.76(0.52-1.12)$ & $0.77(0.52-1.13)$ \\
\hline Per $5-\mathrm{kg} / \mathrm{m}^{2}$ increase & & & $1.17(1.06-1.30)$ & $1.15(1.04-1.27)$ & & & $0.99(0.90-1.09)$ & $0.98(0.89-1.08)$ \\
\hline Height per $10 \mathrm{~cm}^{\mathrm{e}}$ & & & $1.23(1.09-1.39)$ & $1.12(0.98-1.28)$ & & & $1.09(0.99-1.21)$ & $1.10(0.98-1.24)$ \\
\hline
\end{tabular}

${ }^{a}$ Numbers may not sum to total because of missing data.

${ }^{\mathrm{b}}$ From univariate conditional logistic regression model stratified by age.

${ }^{c}$ Adjusted for fracture before 2005 (yes/no), income ( $\leq 7000,>7000-10000,>10000-20000,>20000$ Baht), smoking status (ever/ never), ever-use of alcohol (yes/no), vascular disease (ischemic heart disease or stroke - yes/no), and episodes of purposeful physical activity per week (0, 1-5, $6-10,11-15,>15)$.

${ }^{\mathrm{d} A d d i t i o n a l l y ~ a d j u s t e d ~ f o r ~ e v e r-u s e ~ o f ~ d e p o t ~ m e d r o x y p r o g e s t e r o n e ~ a c e t a t e . ~}$

${ }^{\mathrm{e} A d d i t i o n a l l y ~ a d j u s t e d ~ f o r ~ s e l f-r e p o r t e d ~ w e i g h t ~ i n ~ k i l o g r a m s . ~}$

Table 3. Association between measures of physical activity and fracture incidence among 32339 women and 25865 men in the Thai Cohort Study (2005-2009)

\begin{tabular}{|c|c|c|c|c|c|c|c|c|}
\hline & \multicolumn{4}{|c|}{ Women } & \multicolumn{4}{|c|}{ Men } \\
\hline & $\begin{array}{c}\text { Total } \\
n^{\mathrm{a}}\end{array}$ & $\begin{array}{c}\text { Fracture } \\
n(\%)\end{array}$ & OR $95 \% \mathrm{Cl}^{\mathrm{b}}$ & OR $95 \% \mathrm{Cl}^{\mathrm{c}, \mathrm{d}}$ & $\begin{array}{c}\text { Total } \\
n^{\mathrm{a}}\end{array}$ & $\begin{array}{c}\text { Fracture } \\
n(\%)\end{array}$ & OR $95 \% \mathrm{Cl}^{\mathrm{b}}$ & OR $95 \% \mathrm{Cl}^{\mathrm{C}}$ \\
\hline \multicolumn{9}{|c|}{ Purposeful physical activity (episodes per week) } \\
\hline 0 & 3100 & $72(2.3)$ & 1.00 & 1.00 & 1488 & $58(3.9)$ & 1.00 & 1.00 \\
\hline $1-5$ & 9558 & $228(2.4)$ & $1.04(0.79-1.36)$ & $1.05(0.80-1.38)$ & 5722 & $248(4.3)$ & $0.90(0.67-1.21)$ & $1.12(0.83-1.48)$ \\
\hline $6-10$ & 11066 & $323(2.9)$ & $1.26(0.97-1.64)$ & $1.28(0.98-1.67)$ & 7896 & $327(4.1)$ & $1.01(0.84-1.21)$ & $1.02(0.77-1.35)$ \\
\hline $11-15$ & 4561 & $144(3.2)$ & $1.33(0.99-1.79)$ & $1.34(1.00-1.80)$ & 5072 & $285(5.6)$ & $0.94(0.79-1.11)$ & $1.37(1.02-1.82)$ \\
\hline$>15$ & 3367 & $117(3.5)$ & $1.52(1.12-2.05)$ & $1.52(1.12-2.06)$ & 5201 & $232(4.5)$ & $1.26(1.06-1.51)$ & $1.08(0.80-1.45)$ \\
\hline & & & $P_{\text {trend }}=0.002$ & $P_{\text {trend }}=0.004$ & & & $P_{\text {trend }}=0.3$ & $P_{\text {trend }}=0.4$ \\
\hline Per 5 episodes/week & & & $1.05(1.02-1.08)$ & $1.04(1.01-1.08)$ & & & $1.01(0.99-1.04)$ & $1.01(0.99-1.04)$ \\
\hline \multicolumn{9}{|l|}{ Housework/gardening } \\
\hline Seldom/never & 1106 & $24(2.2)$ & 1.00 & 1.00 & 2114 & $96(4.5)$ & 1.00 & 1.00 \\
\hline $1-3 /$ month & 2468 & $70(2.8)$ & $1.28(0.80-2.06)$ & $1.22(0.76-1.96)$ & 3827 & $178(4.7)$ & $0.98(0.76-1.26)$ & $0.96(0.74-1.24)$ \\
\hline 1-2/week & 8636 & $233(2.7)$ & $1.23(0.80-1.89)$ & $1.17(0.76-1.80)$ & 7142 & $348(4.9)$ & $1.03(0.82-1.29)$ & $0.99(0.78-1.25)$ \\
\hline 3-4/week & 3802 & $85(2.2)$ & $1.06(0.67-1.68)$ & $0.98(0.61-1.56)$ & 3548 & $154(4.3)$ & $0.92(0.71-1.19)$ & $0.86(0.66-1.12)$ \\
\hline Most days & 15968 & $479(3.0)$ & $1.33(0.87-2.02)$ & $1.22(0.80-1.87)$ & 8850 & $376(4.3)$ & $0.93(0.74-1.17)$ & $0.87(0.69-1.10)$ \\
\hline & & & & & & & & \\
\hline \multicolumn{9}{|l|}{ Manual worker ${ }^{\mathrm{e}}$} \\
\hline No & 22896 & $634(2.8)$ & 1.00 & 1.00 & 18160 & $794(4.4)$ & 1.00 & 1.00 \\
\hline Yes & 5355 & $167(3.1)$ & $1.16(0.97-1.39)$ & $1.11(0.92-1.33)$ & 4969 & $258(5.2)$ & $1.15(0.99-1.32)$ & $1.10(0.95-1.28)$ \\
\hline \multicolumn{9}{|l|}{ Sitting time (hrs/day) ${ }^{\mathrm{e}}$} \\
\hline $0-3$ & 8023 & $231(2.9)$ & 1.00 & 1.00 & 7367 & 341 (4.6) & 1.00 & 1.00 \\
\hline $4-7$ & 8953 & $248(2.8)$ & $0.97(0.80-1.17)$ & $0.96(0.80-1.16)$ & 8683 & $418(4.8)$ & $1.04(0.90-1.20)$ & $1.04(0.90-1.20)$ \\
\hline $8-9$ & 5965 & $172(2.9)$ & $1.03(0.84-1.26)$ & $1.04(0.84-1.27)$ & 3816 & $169(4.3)$ & $0.96(0.79-1.15)$ & $0.96(0.79-1.16)$ \\
\hline$\geq 10$ & 8949 & $243(2.7)$ & $0.99(0.82-1.19)$ & $0.99(0.82-1.20)$ & 5624 & $223(4.0)$ & $0.84(0.70-0.99)$ & $0.84(0.71-1.00)$ \\
\hline Per 2 hours & & & $1.00(0.97-1.04)$ & $1.00(0.97-1.04)$ & & & $0.96(0.93-0.99)$ & $0.96(0.93-0.99)$ \\
\hline
\end{tabular}

aNumbers may not sum to total because of missing data.

${ }^{\mathrm{b}}$ From univariate conditional logistic regression model stratified by age.

${ }^{c}$ Adjusted for fracture before 2005 (yes/no), income $(\leq 7000,>7000-10000,>10000-20000,>20000$ Baht), smoking status (ever/never), ever-use of alcohol (yes/no), vascular disease (ischemic heart disease or stroke - yes/no), and body mass index $(<18.5,18.5-<23,23-<24.5,25-<30$, $\left.\geq 30.0 \mathrm{~kg} / \mathrm{m}^{2}\right)$.

${ }^{\mathrm{d} A d d i t i o n a l l y ~ a d j u s t e d ~ f o r ~ e v e r ~ u s e ~ o f ~ d e p o t ~ m e d r o x y p r o g e s t e r o n e ~ a c e t a t e . ~}$

eMultivariable models additionally adjusted for episodes of purposeful physical activity per week $(0,1-5,6-10,11-15,>15)$.

\section{DISCUSSION}

In summary, in this cohort of young and middle-aged Thai adults, the risk of fracture, particularly lower limb fracture, increased with increasing BMI among women. Underweight men appeared to have a lower fracture risk as compared with men with higher BMI values. Among women, frequent purposeful physical activity modestly increased fracture risk at 
Table 4. Association of body mass index (BMI) and measures of physical activity with site-specific fracture incidence among women and men in the Thai Cohort Study

\begin{tabular}{|c|c|c|c|c|c|c|}
\hline & \multicolumn{3}{|c|}{ Women } & \multicolumn{3}{|c|}{ Men } \\
\hline & $\begin{array}{l}\text { Upper limb } \\
(n=137)^{\mathrm{a}}\end{array}$ & $\begin{array}{c}\text { Lower limb } \\
(n=240)^{\mathrm{a}} \\
\text { OR } 95 \% \mathrm{Cl}^{\mathrm{b}, \mathrm{c}}\end{array}$ & $\begin{array}{l}\text { All other sites } \\
\quad(n=535)^{\mathrm{a}}\end{array}$ & $\begin{array}{l}\text { Upper limb } \\
(n=192)^{\mathrm{a}}\end{array}$ & 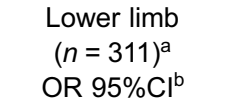 & $\begin{array}{l}\text { All other sites } \\
\qquad(n=694)^{\mathrm{a}}\end{array}$ \\
\hline \multicolumn{7}{|l|}{ BMI $\left(\mathrm{kg} / \mathrm{m}^{2}\right)$} \\
\hline$<18.5$ & $0.76(0.46-1.24)$ & $0.89(0.61-1.30)$ & $1.00(0.79-1.27)$ & $0.44(0.19-1.00)$ & $0.75(0.44-1.29)$ & $0.80(0.55-1.16)$ \\
\hline $18.5-<23.0$ & 1.00 & 1.00 & 1.00 & 1.00 & 1.00 & 1.00 \\
\hline $23.0-<25.0$ & $0.59(0.30-1.19)$ & $1.13(0.73-1.74)$ & $0.89(0.65-1.21)$ & $0.86(0.59-1.25)$ & $0.74(0.54-1.02)$ & $1.02(0.84-1.24)$ \\
\hline $25.0-30.0$ & $0.49(0.21-1.13)$ & $1.40(0.90-2.15)$ & $1.13(0.83-1.54)$ & $0.77(0.51-1.18)$ & $0.97(0.71-1.31)$ & $0.96(0.77-1.18)$ \\
\hline$\geq 30.00$ & $1.50(0.60-3.72)$ & $2.35(1.26-4.37)$ & $1.50(0.92-2.45)$ & $0.65(0.24-1.77)$ & $0.71(0.33-1.52)$ & $0.79(0.48-1.30)$ \\
\hline Per $5-\mathrm{kg} / \mathrm{m}^{2}$ increase & $1.01(0.77-1.34)$ & $1.32(1.10-1.57)$ & $1.10(0.96-1.26)$ & $0.95(0.75-1.20)$ & $0.98(0.82-1.18)$ & $0.99(0.87-1.12)$ \\
\hline \multicolumn{7}{|c|}{ Purposeful physical activity (episodes per week) } \\
\hline 0 & 1.00 & 1.00 & 1.00 & 1.00 & 1.00 & 1.00 \\
\hline $1-5$ & $1.18(0.56-2.47)$ & $0.79(0.48-1.32)$ & $1.16(0.81-1.67)$ & $0.74(0.39-1.39)$ & $1.94(0.97-3.90)$ & $1.04(0.72-1.51)$ \\
\hline $6-10$ & $1.21(0.59-2.52)$ & $1.08(0.69-1.79)$ & $1.38(0.97-1.96)$ & $0.66(0.36-1.22)$ & $1.57(0.79-3.13)$ & $1.03(0.72-1.47)$ \\
\hline $11-15$ & $1.92(0.90-4.13)$ & $1.14(0.67-1.96)$ & $1.29(0.87-1.91)$ & $0.72(0.38-1.37)$ & $2.67(1.34-5.31)$ & $1.27(0.88-1.84)$ \\
\hline \multirow[t]{2}{*}{$>15$} & $1.97(0.89-4.36)$ & $1.26(0.72-2.21)$ & $1.57(1.05-2.35)$ & $0.85(0.46-1.59)$ & $1.69(0.84-3.43)$ & $1.02(0.70-1.49)$ \\
\hline & $P_{\text {trend }}=0.1$ & $P_{\text {trend }}=0.2$ & $P_{\text {trend }}=0.03$ & $P_{\text {trend }}=0.9$ & $P_{\text {trend }}=0.6$ & $P_{\text {trend }}=0.4$ \\
\hline \multicolumn{7}{|l|}{ Sitting time (hrs/day) } \\
\hline $0-3$ & 1.00 & 1.00 & 1.00 & 1.00 & 1.00 & 1.00 \\
\hline $4-7$ & $0.89(0.55-1.44)$ & $0.91(0.64-1.29)$ & $1.01(0.79-1.30)$ & $1.07(0.74-1.55)$ & $0.99(0.75-1.31)$ & $1.11(0.92-1.34)$ \\
\hline $8-9$ & $0.95(0.56-1.63)$ & $0.89(0.60-1.32)$ & $1.13(0.86-1.48)$ & $1.33(0.87-2.07)$ & $0.84(0.58-1.21)$ & $0.94(0.73-1.20)$ \\
\hline$\geq 10$ & $0.91(0.56-1.48)$ & $0.83(0.58-1.20)$ & $1.10(0.86-1.40)$ & $0.92(0.60-1.42)$ & $0.76(0.55-1.07)$ & $0.85(0.68-1.07)$ \\
\hline Per 2-hour increase & $1.00(0.92-1.09)$ & $0.98(0.92-1.05)$ & $1.02(0.98-1.07)$ & $0.98(0.91-1.06)$ & $0.94(0.88-0.99)$ & $0.97(0.93-1.01)$ \\
\hline
\end{tabular}

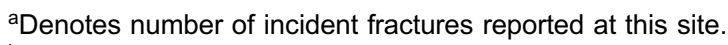

${ }^{b}$ Adjusted for fracture before 2005 (yes/no), income ( $\leq 7000,>7000-10000,>10000-20000,>20000$ Baht), smoking status (ever/ never), ever-use of alcohol (yes/no), vascular disease (ischemic heart disease or stroke - yes/no), kidney disease (yes/no), and episodes of purposeful physical activity per week $(0,1-5,6-10,11-15,>15)$, and BMI $\left(<18.5,18.5-<23,23-<24.5,25-<30, \geq 30.0 \mathrm{~kg} / \mathrm{m}^{2}\right)$.

${ }^{\mathrm{c}}$ Additionally adjusted for ever-use of depot medroxyprogesterone acetate (women only).

all sites, but gardening/housework did not. Among men, greater sitting time was inversely associated with fracture risk independent of purposeful physical activity.

The strengths of this study include the large sample size and prospective nature of the data, although all information, including fracture outcomes, was self-reported. Self-report is a relatively accurate way to obtain information on fracture incidence, ${ }^{30}$ although accuracy varies somewhat by site: arm and leg fractures are well reported but fractures of the hands, feet, and ribs are both under- and over-reported. ${ }^{30,31}$ Fractures that occurred further in the past may have been underreported, which may have attenuated risk estimates, ${ }^{32}$ although repeating the analyses after including only fractures reported for the year before ascertainment did not materially alter the estimates. Over-reporting of height and under-reporting of weight were found to lead to modest BMI misclassification within the Thai Cohort Study, particularly in the overweight and obese categories, ${ }^{33}$ which could attenuate the association between BMI and fracture risk.

It is possible that the $29 \%$ loss to follow-up may have affected our findings. Those who did not complete the second survey were more likely to be single, male, younger, have less prior education, and to have been current smokers or drinkers at baseline, all of which were associated with fracture in our analyses. Physical activity was not related to attrition but obesity was $(32 \%$ of women in the obese II category at baseline did not complete the second survey as compared with $28 \%$ of women in the normal BMI range). However, because reported injury in 2005 was not associated with attrition, there is little reason to expect fractures to be related to participation and, as the models were adjusted for all baseline variables related with attrition, it is unlikely that attrition has materially affected our results. ${ }^{34}$

Participants in the study were a relatively well-educated, ethnically homogeneous group of Thai people, which raises issues of generalizability. Although it is unlikely that the relationship between obesity and fracture varies by ethnicity, the circumstances of fracture occurrence might vary across countries. It may be that mode of fracture (eg, traffic accident vs fall, trauma vs fragility) affected the observed associations; however, we had no information on how fractures occurred. We plan to explore this issue further in subsequent data collections.

BMI and physical activity can positively and negatively influence fracture risk. The overall effect of BMI and physical activity on fracture will therefore be determined by the balance of these factors in an individual. In this cohort, fracture risk was not increased in underweight participants, suggesting that if, on average, underweight reduces bone mineral density in young people in this population, this does not result in more broken bones. Fracture risk was increased in very obese women. Perhaps this increase was mediated by an 
increased likelihood of trauma (falls) and its consequences, and/or the negative effects of severe obesity on bone strength. ${ }^{20}$ There are few data on fracture among younger adults, but in peri-/post-menopausal women higher BMI probably increases risk of ankle fracture but decreases risk of wrist and hip fracture. ${ }^{35}$ Our study had limited power to investigate site-specific effects, but in this young cohort upper limb fracture risk was not increased in underweight women. However, lower limb (leg and ankle) fracture was most strongly associated with high BMI, in keeping with findings in peri-/post-menopausal women. ${ }^{26,35,36}$ It has been suggested that this association is due to (a) the fact that greater body weight increases forces on the leg and ankle during a fall and/ or (b) the increased propensity to fall among obese people, because of greater instability. ${ }^{26,36}$

Among women we found a modest increase in overall fracture incidence with increasing frequency of purposeful physical activity (ie, exercise), although household activity was not associated with risk. This suggests that the physical circumstances in which the activity was done, rather than the exercise per se, contributed to fracture risk in this population. Notably, we found no evidence that any of our physical activity measures were associated with decreased fracture risk among women, suggesting that, even if physical activity increases bone mineral density in this population, it is not of general relevance to fracture risk in this age group. However, our measures did not clearly distinguish between low- and high-impact physical activity and may thus not have captured important discriminating information. ${ }^{37}$

The effects of BMI and physical activity on fracture risk varied between men and women in this population. It is possible that relative differences in fat versus muscle mass partly explain this difference because women tend to have a higher percentage of body fat as compared with men with the same $\mathrm{BMI},{ }^{38}$ and higher fat mass relative to lean body mass can adversely affect bone strength ${ }^{16-20}$ and may increase instability and propensity to fall. ${ }^{21}$ It is also likely that the circumstances in which fractures are sustained vary substantially between sexes. Although we have not explored other factors in depth, we noted that socioeconomic indicators, younger age, and alcohol consumption were significantly associated with fracture occurrence among men, suggesting that risk behaviors may be more important drivers of fracture risk in younger men. Furthermore, the inverse association with sitting time in men may indicate that men who spend more time sitting are less likely to be in physical situations where fracture occurs. These differences warrant further investigation.

In conclusion, our results suggest that, among young women, fracture risk increases with increasing BMI and with frequent purposeful physical activity. The growing prevalence of obesity is therefore likely to increase the overall fracture burden in young women but not in men. Although an active lifestyle has obvious health benefits, particularly for those who are obese, our results highlight the importance of promoting injury prevention practices alongside physical activity recommendations.

\section{ACKNOWLEDGMENTS}

This study was supported by the International Collaborative Research Grants Scheme, with joint grants from the Wellcome Trust UK (WT071587MA) and the Australian National Health and Medical Research Council (NHMRC) (268055 and 585426). SJ is supported by an Early Career Fellowship (Public Health 519796) and EB by a Senior Research Fellowship both from the NHMRC. We thank the staff at Sukhothai Thammathirat Open University (STOU) who assisted with student contact and the STOU students who are participating in the cohort study. We also thank Dr Bandit Thinkamrop and his team from Khon Kaen University for guiding us successfully through the complex data processing.

Conflicts of interest: None declared.

\section{REFERENCES}

1. Barker M, Power C, Roberts I. Injuries and the risk of disability in teenagers and young adults. Arch Dis Child. 1996;75(2): $156-8$.

2. Gore FM, Bloem PJ, Patton GC, Ferguson J, Joseph V, Coffey $\mathrm{C}$, et al. Global burden of disease in young people aged 10-24 years: a systematic analysis. Lancet. 2011;377(9783):2093-102.

3. Key TJ, Appleby PN, Spencer EA, Roddam AW, Neale RE, Allen NE. Calcium, diet and fracture risk: a prospective study of 1898 incident fractures among 34696 British women and men. Public Health Nutr. 2007;10(11):1314-20.

4. Roddam AW, Neale R, Appleby P, Allen NE, Tipper S, Key TJ. Association between plasma 25-hydroxyvitamin D levels and fracture risk: the EPIC-Oxford study. Am J Epidemiol. 2007; 166(11):1327-36.

5. Roddam AW, Appleby P, Neale R, Dowsett M, Folkerd E, Tipper S, et al. Association between endogenous plasma hormone concentrations and fracture risk in men and women: the EPIC-Oxford prospective cohort study. J Bone Miner Metab. 2009;27(4):485-93.

6. De Laet C, Kanis JA, Odén A, Johanson H, Johnell O, Delmas P, et al. Body mass index as a predictor of fracture risk: a metaanalysis. Osteoporos Int. 2005;16(11):1330-8.

7. Karlsson M. Has exercise an antifracture efficacy in women? Scand J Med Sci Sports. 2004;14(1):2-15.

8. MacInnis RJ, Cassar C, Nowson CA, Paton LM, Flicker L, Hopper JL, et al. Determinants of bone density in 30- to 65-yearold women: a co-twin study. J Bone Miner Res. 2003;18(9): 1650-6.

9. Pesonen J, Sirola J, Tuppurainen M, Jurvelin J, Alhava E, Honkanen R, et al. High bone mineral density among perimenopausal women. Osteoporos Int. 2005;16(12):1899-906.

10. Rubin MR, Schussheim DH, Kulak CA, Kurland ES, Rosen CJ, Bilezikian JP, et al. Idiopathic osteoporosis in premenopausal women. Osteoporos Int. 2005;16(5):526-33. 
11. Peris P, Ruiz-Esquide V, Monegal A, Alvarez L, Martínez de Osaba MJ, Martínez-Ferrer A, et al. Idiopathic osteoporosis in premenopausal women. Clinical characteristics and bone remodelling abnormalities. Clin Exp Rheumatol. 2008;26(6): 986-91.

12. Holm K, Dan A, Wilbur J, Li S, Walker J. A longitudinal study of bone density in midlife women. Health Care Women Int. 2002;23(6-7):678-91.

13. Cohen A, Recker RR, Lappe J, Dempster DW, Cremers S, McMahon DJ, et al. Premenopausal women with idiopathic lowtrauma fractures and/or low bone mineral density. Osteoporos Int. 2012;23(1):171-82.

14. Lauder TD, Dixit S, Pezzin LE, Williams MV, Campbell CS, Davis GD. The relation between stress fractures and bone mineral density: evidence from active-duty Army women. Arch Phys Med Rehabil. 2000;81(1):73-9.

15. Winters-Stone KM, Snow CM. Site-specific response of bone to exercise in premenopausal women. Bone. 2006;39(6):1203-9.

16. Weiler HA, Janzen L, Green K, Grabowski J, Seshia MM, Yuen KC. Percent body fat and bone mass in healthy Canadian females 10 to 19 years of age. Bone. 2000;27(2):203-7.

17. Ackerman A, Thornton JC, Wang J, Pierson RN Jr, Horlick M. Sex difference in the effect of puberty on the relationship between fat mass and bone mass in 926 healthy subjects, 6 to 18 years old. Obesity (Silver Spring). 2006;14(5):819-25.

18. Farr JN, Chen Z, Lisse JR, Lohman TG, Going SB. Relationship of total body fat mass to weight-bearing bone volumetric density, geometry, and strength in young girls. Bone. 2010;46(4):977-84.

19. Zillikens MC, Uitterlinden AG, van Leeuwen JP, Berends AL, Henneman P, van Dijk KW, et al. The role of body mass index, insulin, and adiponectin in the relation between fat distribution and bone mineral density. Calcif Tissue Int. 2010;86(2):116-25.

20. Sukumar D, Schlussel Y, Riedt CS, Gordon C, Stahl T, Shapses SA. Obesity alters cortical and trabecular bone density and geometry in women. Osteoporos Int. 2011;22(2):635-45.

21. Winters KM, Snow CM. Body composition predicts bone mineral density and balance in premenopausal women. J Womens Health Gend Based Med. 2000;9(8):865-72.

22. Rocher E, Chappard C, Jaffre C, Benhamou CL, Courteix D. Bone mineral density in prepubertal obese and control children: relation to body weight, lean mass, and fat mass. J Bone Miner Metab. 2008;26(1):73-8.

23. Aekplakorn W, Mo-Suwan L. Prevalence of obesity in Thailand. Obes Rev. 2009;10(6):589-92.

24. Sleigh AC, Seubsman SA, Bain C; Thai Cohort Study Team. Cohort profile: The Thai Cohort of 87,134 Open University students. Int J Epidemiol. 2008;37(2):266-72.

25. World Cancer Resarch Fund/American Institue for Cancer Research. Food, nutrition, physical activity and the prevention of cancer: a global perspective. Washington DC: AICR; 2007.

26. Honkanen R, Tuppurainen M, Kröger H, Alhava E, Saarikoski S.
Relationships between risk factors and fractures differ by type of fracture: a population-based study of 12192 perimenopausal women. Osteoporos Int. 1998;8(1):25-31.

27. Moran DS, Israeli E, Evans RK, Yanovich R, Constantini N, Shabshin N, et al. Prediction model for stress fracture in young female recruits during basic training. Med Sci Sports Exerc. 2008;40(11 Suppl):S636-44.

28. Välimäki VV, Alfthan H, Lehmuskallio E, Löyttyniemi E, Sahi $\mathrm{T}$, Suominen $\mathrm{H}$, et al. Risk factors for clinical stress fractures in male military recruits: a prospective cohort study. Bone. 2005; 37(2):267-73.

29. Banwell C, Lim L, Seubsman SA, Bain C, Dixon J, Sleigh A. Body mass index and health-related behaviours in a national cohort of 87,134 Thai open university students. J Epidemiol Community Health. 2009;63(5):366-72.

30. Honkanen K, Honkanen R, Heikkinen L, Kröger H, Saarikoski S. Validity of self-reports of fractures in perimenopausal women. Am J Epidemiol. 1999;150(5):511-6.

31. Ismail AA, O'Neill TW, Cockerill W, Finn JD, Cannata JB, Hoszowski K, et al. Validity of self-report of fractures: results from a prospective study in men and women across Europe. EPOS Study Group. European Prospective Osteoporosis Study Group. Osteoporos Int. 2000;11(3):248-54.

32. Berecki-Gisolf J, McClure R, Seubsman SA, Sleigh A; Thai Cohort Study team. Reporting of lifetime fractures: methodological considerations and results from the Thai Cohort Study. BMJ Open. 2012;2(4).

33. Lim LL, Seubsman SA, Sleigh A. Validity of self-reported weight, height, and body mass index among university students in Thailand: Implications for population studies of obesity in developing countries. Popul Health Metr. 2009;7:15.

34. Spratt M, Carpenter J, Sterne JA, Carlin JB, Heron J, Henderson $\mathrm{J}$, et al. Strategies for multiple imputation in longitudinal studies. Am J Epidemiol. 2010;172(4):478-87.

35. Holmberg AH, Johnell O, Nilsson PM, Nilsson J, Berglund G, Akesson K. Risk factors for fragility fracture in middle age. A prospective population-based study of 33,000 men and women. Osteoporos Int. 2006;17(7):1065-77.

36. Valtola A, Honkanen R, Kröger H, Tuppurainen M, Saarikoski $\mathrm{S}$, Alhava E. Lifestyle and other factors predict ankle fractures in perimenopausal women: a population-based prospective cohort study. Bone. 2002;30(1):238-42.

37. Martyn-St James M, Carroll S. Effects of different impact exercise modalities on bone mineral density in premenopausal women: a meta-analysis. J Bone Miner Metab. 2010;28(3): 251-67.

38. Heo M, Faith MS, Pietrobelli A, Heymsfield SB. Percentage of body fat cutoffs by sex, age, and race-ethnicity in the US adult population from NHANES 1999-2004. Am J Clin Nutr. 2012; 95(3):594-602. 